\title{
Investigación en Progreso: Gestión de la Educación Superior en Contextos de Masividad Basada en Tecnologías Inteligentes de Transformación de Información
}

\author{
Laura Cecilia Diaz ${ }^{1,2}$ \\ ${ }^{1}$ Universidad Nacional de Córdoba. Córdoba Capital, Argentina \\ ${ }^{2}$ Grupo de Investigación en Sistemas de Información. Universidad Nacional de Lanús \\ Remedios de Escalada, Argentina \\ lcd_ic@yahoo.com.ar
}

\begin{abstract}
Resumen- El propósito de esta investigación -tesis actualmente en desarrrollo para la Carrera de Doctorado en Administración y Política Públicas de la Universidad Nacional de Córdoba- es contribuir a facilitar la apropiación del conocimiento en Educación Superior en contextos de masividad. Se espera que esta contribución resulte novedosa, tanto en las interpretaciones sobre la información del estudiante que emerjan de las acciones previstas, como en la construcción de un diseño metodológico aplicable en las muy diversas prácticas de este escenario. La propuesta considera el uso de Tecnologías Inteligentes de Explotación de la Información y otras herramientas del campo de la Inteligencia Computacional para construir categorías y enriquecer teorías a la luz del Paradigma Interpretativo de investigación en las Ciencias Sociales. En particular, a partir de la experiencia piloto, se proporcionará un mejor conocimiento del estudiante de cursos masivos de esta Universidad en sus características socio-económicas y de rendimiento académico.
\end{abstract}

Palabras Clave- Educación Superior, Apropiación del conocimiento, Tecnologías Inteligentes de Explotación de la Información, Paradigma Interpretativo de las Ciencias Sociales.

\section{JUSTIFICACIÓN DE LA PROPUESTA Y ESTADO DEL ARTE}

Múltiples son los aspectos que despiertan el interés por realizar aportes para la Gestión de la Educación Superior en estos tiempos en que la construcción de saberes constituye un desafío importante [1].

Un aspecto de singular relevancia es el enfoque hacia los contextos de masividad. Actualmente es posible pensar que un ciudadano de un continente cualquiera puede tener acceso a un nivel de acreditación de postgrado de Universidades reconocidas en el mundo entero. Este fenómeno actual surge como factible a partir de la incorporación de las Tecnologías de Información y Comunicación (TICs) en los procesos de aprendizaje y evaluación.

Proveer de información para un adecuado diseño de las políticas públicas en Educación Superior, despierta el interés para contribuir con una mejor apropiación del conocimiento por parte de la sociedad. En esta dirección, una dimensión relevante es la asociada a las características del estudiante, principal actor de este complejo escenario.

El uso de Inteligencia Computacional y de las TIEI, ofrece la oportunidad de descubrir comportamientos socioeconómicos, académicos, cognitivos, entre otros. de los sujetos en procesos de aprendizaje, que con otras metodologías no serían necesariamente detectados [2].

Otro tema relevante, que las agendas políticas incluyen cada vez con mayor presencia es la mejora, tanto en cantidad como en calidad, de profesionales egresados de las carreras denominadas TICs, en particular de las Ingenierías. Ello atento a satisfacer la demanda de especialistas en estas tecnologías. Tal es el caso de la Agencia Nacional de Promoción Científica y Tecnológica dependiente del Ministerio de Ciencia, Tecnología e Innovación Productiva de la Nación.

Desde los actores directamente involucrados, la incorporación de técnicas de Inteligencia Artificial en el uso de TICs para la enseñanza de contenidos curriculares de estas carreras en diversas Universidades del mundo, ha dado muestras de mejora tanto en los procesos de aprendizaje como en los de acreditación [3-5].

Además, la detección temprana de las capacidades de los estudiantes resultó un factor significativo en la mejora del aprendizaje de Programación, estos hallazgos realizados en [6], despiertan el interés en el uso de Sistemas de Redes Neuronales Artificiales y otras Tecnologías Inteligentes para predecir su rendimiento académico en las carreras de Ingeniería.

Las acciones llevadas a cabo por el proyecto de investigación: "Sistemas Inteligentes Aplicados a la Enseñanza de Programación en Ingeniería" (SIAEPI) 2012-2013, acreditado por la SECyT de la UNC, se orientaron en esa dirección. Los resultados mostraron una mejora en el rendimiento académico de los estudiantes de la asignatura Informática cuando se retroalimentaba los procesos de evaluación con información detectada por expertos [7].

En razón de la información disponible y de la importancia de los contextos de masividad que promueven una mayor apropiación del conocimiento, el propósito de esta propuesta es contribuir con un diseño metodológico para la obtención de la información relativa al estudiante para la gestión de la Educación Superior en esos contextos, específicamente de la UNC, tomando como casos de estudio dos cursos masivos cuya información está accesible: Informática de la Facultad de Ciencias Exactas, Físicas y Naturales (FCEFyN) y Administración y Sistemas de Información Gubernamental de la Facultad de Ciencias Económicas (FCE). Se espera lograr un mejor conocimiento de las características del estudiante, protagonista principal de este escenario. 
Para el primer caso escogido, perteneciente al grupo de carreras de las denominadas de interés prioritario por el Ministerio de Educación de la Nación, se espera además enriquecer la comprensión del fenómeno de mejora en el rendimiento académico acontecido a partir de los procesos desarrollados en el proyecto de investigación SIAEPI durante el periodo 2012-2013. Esto implicará la descripción de las percepciones que los estudiantes tienen acerca de los procesos de retroalimentación incorporados en sus evaluaciones y del significado que le dan a esas prácticas.

\section{A. La Contribución De La Inteligencia Artificial}

Con el advenimiento del computador, los esfuerzos del campo de La Inteligencia Artificial (IA) se enfocan a lograr la comprensión de entidades inteligentes [8]. Dentro de este vasto campo, merecen especial atención a los fines de esta propuesta de investigación, las Tecnologías Inteligentes de Explotación de la Información. Así por ejemplo, los Sistemas Neuronales Artificiales implementados en computadoras, inspirados en el comportamiento del cerebro humano, aprenden a partir de un importante número de conexiones de neuronas artificiales, las cuales son dispositivos inteligentes cuyo funcionamiento es muy básico. Simulan los procesos de sinapsis y, a partir de ellos, aprenden de los ejemplos que se les proporciona durante las instancias de entrenamiento para adquirir un estado tal que los transforme en Sistemas capaces de responder a nuevas situaciones [9].

Estas técnicas del campo de la IA proporcionan nuevas metodologías para el abordaje de problemas de las ciencias sociales, siendo capaces de predecir, detectar, relacionar, asociar (entre otros), en contextos caracterizados por el gran volumen de información, la complejidad y la incertidumbre. Los antecedentes en el ámbito de la Educación Superior, inspiradores de esta propuesta de trabajo son:

La identificación de las características socioeconómicas y demográficas más relevantes para construir un modelo de comportamiento académico de los estudiantes, como información para el diseño de políticas públicas en Educación Superior. Se hallaron estudios realizados para la Universidad Nacional de Misiones [2] y para la Universidad Nacional de San Juan [10] .

Los Sistemas Tutores Inteligentes (STI) aplicados para atender los problemas de aprendizaje en carreras TICs. Ellos permiten establecer una adecuada realimentación semántica así como un medio para poner en evidencia el conflicto cognitivo, usando como base para su desarrollo los modelos cognitivos [11]. Entre los STI desarrollados específicamente para los cursos de programación que ponen de manifiesto los diferentes modelos conceptuales que les sirven de base. Las arquitecturas de los STI Se describen en [12] y la edición de 2007 de la revista de la IEEE Intelligent Systems se dedica a los STI describiendo un amplio panorama [13].

\section{B. El Paradigma Interpretativo y los procesos de TIEI}

Las TIEI y otras tecnologías del campo de la Inteligencia Computacional, de alguna manera se asemejan a los procesos del paradigma interpretativo de las ciencias sociales, en el sentido que son procesos de descubrimiento a partir de los datos 'sin teorías o supuestos a priori'. A partir de la aplicación de procesos tales como Arboles de inducción, Redes Neuronales Auto Organizadas y Redes Bayesianas, descubren reglas, relaciones, atributos o características que posibilitan indagar, explicar, comprender o describir fenómenos o sucesos, entre otros. En este universo, las Redes Neuronales
Auto Organizadas buscan entre una gran masa de datos de diferentes atributos de 'los individuos' objeto de investigación, las similitudes existentes entre ellos para agruparlos luego en categorías cuyas características son desconocidas a priori. No poseen relaciones causales y la cantidad y tamaño de los agrupamientos son descubiertos al finalizar el procesamiento computacional. No detectan causalidades, sólo relaciones de similitud. Los criterios de similitud no responden al problema abordado sino a las definiciones de distancias computacionales, es decir pertenecen al campo de lo sub-simbólico [8].

Desde la metodología de la Inteligencia Computacional la única validez son los datos disponibles y las interpretaciones que de ellos se hagan dependen del investigador.

Esta actividad de interpretar los resultados depende del marco teórico adoptado, de la postura epistemológica y de la actitud para el 'descubrimiento'. Esta es la primera hermenéutica: indagar, en base los resultados obtenidos mediante esas tecnologías inteligentes, para construir categorías, modelos y teorías a partir de interpretaciones realizadas desde la epistemología del sujeto cognoscente. En este sentido, la Teoría fundamentada se aprecia como metodología adecuada: "La teoría fundamentada no es una teoría, sino una metodología para descubrir teorías que dormitan en los datos" [14].

En las interpretaciones desde la perspectiva de los agentes sociales, se asume epistemológicamente la complejidad del fenómeno social y al investigador inmerso en él "es posible utilizar este diseño en investigaciones cuantitativas que, utilizando datos secundarios -obtenidos en forma rigurosa-, se propongan crear teoría y nuevos conceptos, analizándolos en forma libre inductiva" [15].

En relación con los supuestos axiológicos, la reflexividad puesta de manifiesto en la capacidad del investigador para interpretar tanto los resultados de los procesos de TIEI como los hallazgos de las indagaciones de los agentes sociales, juegan un rol de relevancia.

\section{OBJETIVOS Y PREGUNTAS}

El vacío de conocimiento surge cuando se intenta interpretar desde los diversos actores del escenario de la ES en contextos de masividad y para los propósitos expresados, los resultados obtenidos en los procesos de TIEI alrededor de la información relativa al estudiante.

Los interrogantes que guían esta propuesta de investigación son:

¿Cómo se vinculan y articulan los procesos de TIEI con el análisis desde el paradigma interpretativo en la exploración de un diseño metodológico para la Gestión de la Educación Superior en contextos de masividad?

\section{En ese sentido:}

¿Cómo se caracteriza la respuesta académica de los estudiantes de la UNC, en relación con variables socioeconómicas, usando procesos de TIEI?

¿Cómo perciben esta información los diversos actores involucrados de la UNC y cómo la vinculan a sus prácticas?

¿Cómo significa el estudiante la información descubierta y cómo afecta en los procesos de apropiación del conocimiento?

¿Cómo los estudiantes de Informática en Ingeniería de la FCEFyN de la UNC, perciben la experiencia de retroalimentación en sus procesos de evaluación?.

\section{A. Objetivo General}

Desarrollar un diseño metodológico que permita comprender las características significativas del estudiante, 
como actor principal en Educación Superior en contextos de masividad con el propósito de facilitar la apropiación del conocimiento.

Estas características, en razón de su importancia y disponibilidad de datos, serán abordadas desde las dimensiones socioeconómicas y académicas para dos casos de estudio de la UNC.

\section{B. Objetivos Específicos}

Detectar características socioeconómicas de los estudiantes de asignaturas en contextos de masividad en la UNC, en relación a su rendimiento académico: FCEFyN y FCE de la $\mathrm{UNC}$.

Comprender como significan los protagonistas del escenario de la gestión de la Educación Superior en la UNC, la información obtenida con el uso de TIEI referida a los estudiantes.

Conocer cómo perciben los estudiantes sus procesos cognitivos a partir de la información detectada en los procesos de TIEI.

Comprender cómo influencia en sus procesos de aprendizaje, la retroalimentación realizada en las evaluaciones.

\section{ASPECTOS METODOLOGICOS}

El diseño metodológico propone tres momentos, los dos últimos se desarrollarán simultáneamente, interactuando para tomar decisiones del curso de la investigación: el primero se abocará a una investigación documental de tipo exploratoria, en el segundo se desarrollarán procesos de Tecnologías Inteligentes y el tercero abordará el problema desde el paradigma interpretativo, tomando como propuesta tentativa a La Teoría Fundamentada para construir categorías a partir de los datos y analizar relaciones relevantes entre ellos para permitir que emerja la teoría.

Se enriquecerán las categorías que se detecten a partir de los procesos de TIEI, permitiéndose descubrir nuevas $\mathrm{y}$ modificar las existentes a la luz de las interpretaciones hechas por los agentes sociales indagados y los significados que ellos les otorgan.

Se indagará a actores directamente involucrados con el diseño de políticas en diferentes ámbitos de la UNC, para contextos de masividad. Además, a estudiantes de ambos casos de estudio, sus actitudes y aptitudes para el aprendizaje y el rendimiento académico. Por otra parte, el análisis realizado por expertos mediante un proceso inductivo (2012-2013), que detectaron las semánticas de los estudiantes, se propone como la ontología emergente de la teoría sustantiva.

\section{IMPORTANCIA DEL PROYECTO - AVANCES}

La concreción de este proyecto proporcionará contribuciones novedosas en dos sentidos: $1^{\circ}$ un diseño metodológico para el tratamiento de la información en la Gestión de la Educación Superior en contextos de masividad y $2^{\circ}$ conocimiento de aspectos relativos al estudiante que quedarán disponibles para ser utilizadas en las muy diversas prácticas de este escenario, tales como: el diseño de políticas públicas, los modelos del estudiante en Sistemas Tutores Inteligentes, el diseño de MOOC y, la detección temprana de capacidades para facilitar acciones preventivas y correctivas en los procesos de aprendizaje y evaluación, entre otros.

Durante los periodos 2012-2013 se realizaron avances en la implementación a la asignatura Informática de las carreras de Ingeniería de la FCEFyN de la UNC. Algunos resultados se muestran en [7].
Se escogieron STI disponibles como servicios web y otros que se pueden instalar en la plataforma Moodle, SIETTE [16] .

Por otra parte, se usaron tecnologías inteligentes para el descubrimiento de patrones de comportamiento en la detección temprana de dificultades cognitivas del estudiante. Algunos resultados de los experimentos en [17] .

Los conocimientos que surgen de esta experiencia piloto permiten una plataforma inicial a partir de la cual es posible construir categorías atendiendo a la epistemología del sujeto cognoscente desde el paradigma interpretativo de investigación en las ciencias sociales.

\section{CONDICIONES INSTITUCIONALES}

$\mathrm{Si}$ bien los aportes principales de este trabajo de investigación están orientados a la Universidad Nacional de Córdoba, Institución a la que pertenece la autora; el desarrollo de la tesis doctoral está radicado en el Grupo de Investigación en Sistemas de Información de la Universidad Nacional de Lanus.

\section{REFERENCIAS}

[1] M. Zabalza, «Presentación Acto Inaugural,» de VIII Congreso Iberoamericano de Docencia Universitaria y Nivel Superior, Rosario, 2014.

[2] H. Kuna, García Martinez R. y R. Villatoro, «Identificación de causales de abandono de esstudios universitarios. Uso de procesos de explotación de información,» Revista Iberoamericana de Tecnología en Educación y Educación en Tecnología. TE\&ET, p. Art. 5, 2010.

[3] A. Mitrovic, B. Martín y P. Surawera, «Intelligent Tutors for All: The Constraint-Based Approach,» IEEE Intelligent Systems, vol. 22, nº 4, 2007.

[4] M. desJardins, A. Ciavolino y R. Deloatch, «Playing to Program: Towards an Intelligent Programming Tutor for RURPLE,» Association for the Advance of Artificial Intelligence, 2011.

[5] A. Yadin, «Reducing the Dropout Rate in Introductory Programming Course,» ACM Inroads, vol. 2, nº 4, 2011.

[6] R. Bornat, S. Dehnadi y S. Hamilton, «Mental models, Consistency, and Programming Aptitude,» Australian Computer Society. ACE 2008, 2008.

[7] L. Díaz y C. Bartó, «Tutores virtuales inteligentes: una estrategia de mejora del rendimiento académico en Ingeniería,» de $6^{\circ}$ Seminario Internacional de Educación a Distancia, Mendoza, 2013.

[8] S. Russell y P. Norvig, Inteligencia Artificial. Un enfoque moderno, México: Prentice Hall Hispanoamericana, SA, 1995.

[9] B. Martín del Brío y A. Sanz, Redes Neuronales y Sistemas Difusos. $2^{\circ}$ Edición, Colombia: Alfaomega Grupo Editor, 2005.

[10] S. Formia, L. Lanzarini y W. Hasperué, «Caracterización de la deserción universitaria en la UNRN utilizando Minería de Datos. Un caso de estudio,» Iberoamericana de Educación en Tecnología y Tecnología en Educación (TE\&ET), n 11, 2013.

[11] Koedinger y R. Kenneth, «Cognitive Tutors as Modeling Tools and Instructional Models. Cap. 5,» de Smart Machines in Education, AAAI Press / MIT Press, 2001.

[12] Z. Cataldi, F. Salgueiro y R. Garcia Martinez, «Sistemas Tutores Inteligentes: Los estilos del estudiante para selección de tutorado,» Escuela de Postgrado. ITBA, 2010.

[13] L. Aroyo, A. Graesser y L. Johnson, «Guest Editors' Introduction: Intelligent Educational Systems of the Present and Future,» IEEE Intelligent Systems, vol. 2, nº 4, 2007.

[14] A. Strauss, «En conversación con Heiner Legewie y colaboradores,» Forum Qualitative Social Researchs, vol. Online Journal, p. Art. 22, 2004. 
[15] Mendizabal N., «Los Componentes del diseño flexible en la investigación cualitativa,» de Estrategias de investigación cualitativa. Vasilachis I., Barcelona, Gedisa, 2006.

[16] E. Guzmán, R. Conejo y J. Pérez de la Cruz, «Improving Student Performance Using Self-Assessment Tests,» IEEE Intelligent Systems, vol. 22, no 4, 2007.

[17] L. Díaz, A. Algorry, M. Eschoyez, R. Marangunic y C. Bartó, «Actions Towards the Application of Intelligent Systems in Computer Education,» IEEE LATIN AMERICAN TRANSACTIONS, vol. 11, 2013.

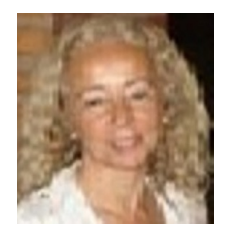

Laura Cecilia Díaz, es Ingeniera Civil y Magister en Administración Pública con orientación en Gestión Gubernamental, ambos de la Universidad Nacional de Córdoba (UNC). Además, Especialista en Ingeniería en Calidad de la Universidad Tecnológica Nacional. Ha publicado en diversas temáticas relacionadas con la Enseñanza en las carreras de Ingeniería, la Gestión de la Calidad y la Inteligencia Artificial. Ha dictado y dicta cursos de Post-grado. Es Profesor dedicación exclusiva y Directora de Proyecto de Investigación en la Facultad de Ciencias Exactas, Físicas y Naturales de la UNC. Es Alumna del Programa el Doctorado en Administración y Política Pública de la UNC e Investigadora Tesista Adscripta del Grupo de Investigación en Sistemas de Información. Universidad Nacional de Lanús. Sus temas actuales abordan la incorporación de herramientas de Inteligencia Artificial en la Gestión Pública y en la Educación Superior en contextos de masividad. 